\title{
Antioxidant Activity of NADH and Its Analogue - An In Vitro Study
}

\author{
Robert Antoni Olek, Wieslaw Ziolkowski, Jan Jacek Kaczor, Lucedio Greci ${ }^{\dagger}$, \\ Jerzy Popinigis and Jedrzej Antosiewicz* \\ Department of Bioenergetics, Jedrzej Sniadecki Academy, School of Physical Education and Sport, Wiejska 1, 80-336 Gdansk, Poland \\ 'Dipartimento di Scienze dei Materiali e della Terra, via Brecce Bianche, University of Ancona, I-60131 Ancona, Italy
}

Received 1 July 2003, Accepted 17 October 2003

\begin{abstract}
The antioxidant activities of NADH and of its analogue, 1,4-dihydro-2,6-dimethyl-3,5-dicarbethoxy-pyridine ( $\left.\mathrm{PyH}_{2}\right)$, were evaluated in vitro. NADH was found to be oxidized by the peroxyl radical derived from 2,2-azobis-(2amidinopropane) dihydrochloride (AAPH) decomposition, in a pH-dependent manner. Both NADH and $\mathbf{P y H}_{2}$ inhibited the peroxidation of egg yolk lecithin (EYL) liposomes, although $\mathrm{PyH}_{2}$ was more effective than $\mathrm{NADH}$ when 2,2'-azobis-4-methoxy-2,4-dimethyl-valeronitrile (methoxyAMVN) was employed to induce EYL liposome peroxidation. The antioxidant activities of $\mathrm{NADH}$ and $\mathrm{PyH}_{2}$ were also evaluated by measuring their influences on 1,3diphenylisobenzofuran (DPBF) fluorescence decay in the presence of peroxyl radicals. NADH and $\mathrm{PyH}_{2}$ were much more effective at inhibiting DPBF quenching in Triton $\mathrm{X}$ 100 micelles than in liposomes. These results indicate that NADH can inhibit lipid peroxidation despite being hydrophilic. Nevertheless, membrane penetration is an important factor and limits its antioxidant activity.
\end{abstract}

Keywords: Antioxidants, Lipid peroxidation, NADH oxidation

\section{Introduction}

Nicotinamide nucleotides are involved in many enzymatic reactions and may act as hydrogen donors in their reduced forms, or as hydrogen acceptors in their oxidized forms (You, 1985). In addition, other studies have shown that these

Abbreviations: NADH, nicotinamide adenine dinucleotide reduced form; EYL, egg yolk lecithin; SOD, superoxide dismutase; $\mathrm{PyH}_{2}, 1,4-$ dihydro-2,6-dimethyl-3,5-dicarbethoxy-pyridine; methoxy-AMVN, 2,2'azobis-(4-methoxy-2,4-dimethyl-valeronitrile); AAPH, 2,2-azobis-(2amidinopropane) dihydrochloride; DPBF, 1,3-diphenylisobenzofuran; TEMPOL, 4-hydroxy-2,2,6,6-tetramethylpiperidine-1-oxyl.

*To whom correspondence should be addressed.

Tel: +48-58-5547325; Fax: +48-58-5520751

E-mail: jant@awf.gda.pl coenzymes can react directly with free radicals (Chan and Bielski, 1974; Bodaness and Chan, 1977; Chan and Bielski, 1980; Carlson et al., 1984; Forni and Willson, 1986a, 1986b). Years ago it was observed that NADH reacts only slowly with superoxide radical-anions and that this reaction proceeds faster when NADH is bound to lactate dehydrogenase (Chan and Bielski, 1974) or to glyceraldehyde-3-phosphate dehydrogenase (Chan and Bielski, 1980). It has also been demonstrated that NADH efficiently reacts with several radicals via hydrogen transfer. For example, the rate constant of the reaction between the promethazine radical cation and $\mathrm{NADH}$ is $3.2 \times 10^{7} \mathrm{M}^{-1} \mathrm{~s}^{-1}$, and that those of reactions between radicals such as the promazine radical cation or benzoquinone fall in the same range (Carlson et al., 1984). It has also been reported that thiyl radicals react directly with $\mathrm{NADH}$ with rate constants of $5.8 \times 10^{8} \mathrm{M}^{-1} \mathrm{~s}^{-1}$ and $2.3 \times 10^{8} \mathrm{M}^{-1} \mathrm{~s}^{-1}$ for cysteine and glutathion, respectively (Forni and Willson, 1986a, 1986b). The oxidation of NADPH by singlet oxygen has also been described (Bodaness and Chan, 1977). All of these examples are based on in vitro experiments because of the complexity of the systems in which these coenzymes function pose difficulties with respect to demonstrating whether they react with free radicals in vivo.

It is intriguing that NADH and NADPH concentrations in living cells are relatively high. For example, in hepatocytes mitochondrial and cytoplasmic NADH concentrations have been calculated to be $0.64 \mathrm{mM}$ and $0.27 \mathrm{mM}$, respectively (Tischler et al., 1977). Furthermore, these can be easily modulated by changes in metabolic rate. For example, a threefold increase in the cytosolic NADH content was reported in skeletal muscle after intensive exercise (Schantz, 1986). Moreover, a significant increase in hepatocyte NADH content was observed after ethanol treatment (Lieber, 1997).

The high reactivity of NADH with some free radicals and its high intracellular concentrations led us to ponder the question as whether NADH should be considered an antioxidant in biological systems. In order to obtain an answer to this question, the influences of NADH and of its analogue, $\mathrm{PyH}_{2}$, on lipid peroxidation were investigated. 


\section{Materials and Methods}

NADH oxidation by peroxyl radicals NADH oxidation was measured by determining its reduced absorbancy at $340 \mathrm{~nm}$, with a CECIL CE 9200 spectrophotometer. The peroxyl radicals were generated by decomposing $30 \mathrm{mM}$ of 2,2'-azobis-(2amidinopropane) dihydrochloride (AAPH) at $37^{\circ} \mathrm{C}$, as previously described (Niki, 1990). Experiments were carried out at pH 7.4 and 6.3 in $50 \mathrm{mM}$ phosphate buffer ( $2 \mathrm{ml}$ final volume) in the presence or absence of $1 \mathrm{mM}$ 4-hydroxy-2,2,6,6-tetramethylpiperidine-1oxyl (TEMPOL).

Lipid peroxidation of egg yolk lecithin (EYL) liposomes EYL liposomes were prepared according to the method of Batzri and Korn (1973). Five mg of EYL liposomes were oxidized in a final

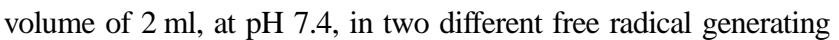
systems. The first systeminvolved on the reaction between cytochrome c $(4.2 \mu \mathrm{M})$ and $3.6 \mathrm{mM}$ of tert-butyl hydroperoxide $(\mathrm{Bu} \mathrm{tOOH})$, whereas the second involved the use of the products of $1 \mathrm{mM}$ 2,2'-azobis-(4-methoxy-2,4-dimethyl-valeronitrile) (methoxyAMVN) thermal decomposition at $37^{\circ} \mathrm{C}$. The consumption of oxygen was measured using a Gilson Oxygraph equipped with a Clark electrode, and the rate of oxygen consumption was used as an indicator of lipid peroxidation.

\section{Measurement of DPBF fluorescence quenched by free radicals EYL liposomes or Triton micelles were treated for $10 \mathrm{~min}$, with $10 \mathrm{ml}$ or $2 \mu \mathrm{l}$ of $2 \mathrm{mM} \mathrm{1,3-diphenylisobenzofuran} \mathrm{(DPBF)} \mathrm{ethanol}$ solution, respectively, and emission at $455 \mathrm{~nm}$ was recorded at an excitation wavelength of $410 \mathrm{~nm}$ (Wozniak et al., 1991; Wozniak et al., 1993; Tanfani et al., 1994) using a Perkin Elmer spectrofluorometer LS5. DPBF fluorescence quenching was obtained using $6 \mathrm{mM}$ AAPH. The same procedure was performed in the presence of NADH or of its analogue, $\mathrm{PyH}_{2}$. \\ All results are the averages of at least five measurements, and values are expressed as means $\pm \mathrm{SD}$.}

Materials 2,2'-azobis-(2-amidinopropane) dihydrochloride (AAPH), tert-butyl hydroperoxide and 1,3-diphenylisobenzofuran (DPBF) were from Aldrich Chemical Co. (Milwaukee, USA), 5,5'Dithiobis(2-nitrobenzoic acid (DTNB), and 2,2'-azobis-4-methoxy2,4-dimethyl-valeronitrile (methoxy-AMVN) was purchased from Wako Pure Chemical Industries Ltd. (Osaka, Japan). All other reagents were purchased from Sigma Chemical Co. (St. Louis, USA) and were of the purest grade available.

\section{Results and Discussion}

NADH oxidation by peroxyls at different pHs Peroxyl radicals are intermediates in lipid (Brault et al., 1985) and protein (Gieseg et al., 2000) peroxidation. Although much evidence indicates that NADH can react with several types of free radicals (Chan and Bielski, 1974; Bodaness and Chan, 1977; Chan and Bielski, 1980; Carlson et al., 1984; Forni and Willson, 1986a, 1986b), no data is available concerning NADH reactivity towards peroxyl radicals. In this experimental model, we used peroxyl radicals generated from AAPH. Our results show that a peroxyl radical generated by AAPH is able to promote hydrogen transfer from NADH, and that this leads to the formation of hydroperoxide and a NAD radical, which may then react with dioxygen to form a superoxide radical anion $\left(\mathrm{O}_{2}^{-}\right)$(Chan and Bielski, 1974). Experiments were performed at $\mathrm{pH} 7.4$ because of its physiologic relevance or at 6.3, which is found in skeletal muscle after intensive exercise (Sahlin et al., 1976). We found that the reaction between $\mathrm{NADH}$ and peroxyl radicals proceeds faster at a lower $\mathrm{pH}$ (Fig. 1). As reported previously, AAPH decomposition mainly depends on temperature and only to a minor extent on $\mathrm{pH}$ (Niki, 1990). We observed ca. 12\% higher NADH oxidation at $\mathrm{pH} 6.3$ vs. $\mathrm{pH}$ 7.4. When oxygen consumption by AAPH solution at $37^{\circ} \mathrm{C}$ was measured, which directly what reflects AAPH decomposition, 5\% lower oxygen consumption was

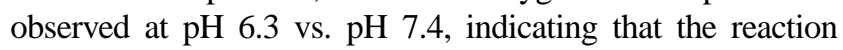
between NADH and peroxyl radical is accelerated at lower pHs. In addition, the rate of NADH oxidation by AAPH increased with AAPH concentration, in a NADH concentration in dependent manner (data not shown). As reported previously, AAPH decomposes into a carbon centered radical and then into a peroxyl radical, at a constant rate during the first few hours of incubation at $37^{\circ} \mathrm{C}$ (Niki, 1990). Thus, we assumed that the oxidation of $\mathrm{NADH}$ was carried out by a constant quantity of peroxyl radical and that thus the NADH concentration had no influence on the oxidation process. TEMPOL (Fig. 1), but not SOD (data not shown), reduced the rate of NADH oxidation mediated by AAPH. TEMPOL reacts with superoxides or with carbon-centered radicals (Samuni et al., 1990) but does not react with peroxyl and alkoxyl radicals (Chateauneuf et al., 1988). The lack of effect by SOD indicates that $\mathrm{O}_{2}^{-}$and its protonated form, the hydroperoxyl radical, play no role in NADH oxidation. However, the inhibitory effect of TEMPOL implies that the carbon center radical is also involved in the oxidation of NADH. The data obtained shows that NADH oxidation can proceed by either peroxyl or carbon centered radicals generated from AAPH. The stimulating effect of $\mathrm{pH} 6.3$ on NADH oxidation almost disappeared in the presence of TEMPOL (Fig. 1). This can be explained firstly by the fact that TEMPOL antioxidant activity is higher at lower $\mathrm{pH}$, and secondly because NADH oxidation is $\mathrm{pH}$ dependent only for specific radicals species. We believe that the first postulated mechanism is the most probably as NADH is a $\mathrm{pH}$ sensitive compound and easily decomposes in acids.

The lag phase observe during NADH oxidation can be completely eliminated if AAPH is incubated for at least $20 \mathrm{~min}$ at $37^{\circ} \mathrm{C}$ before $\mathrm{NADH}$ is added (data not shown), under these circumstances NADH oxidation proceeds at a constant rate (Fig. 1). This result indicates that some time is necessary for the radicals in AAPH solution to reach a constant concentration.

Protective effect of $\mathrm{NADH}$ and $\mathrm{PyH}_{2}$ on lipid peroxidation Since NADH reacts with two types of free radicals (the 


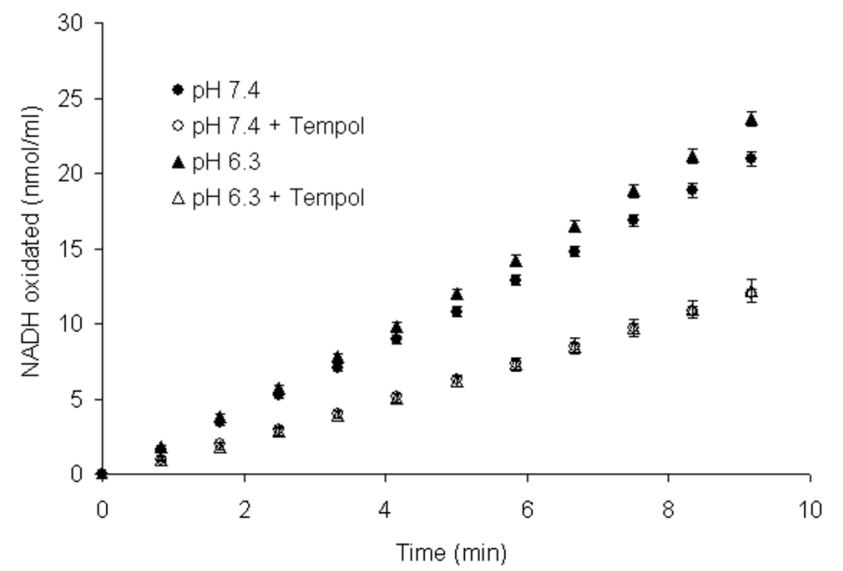

Fig. 1. Effects of $\mathrm{pH}$ on $\mathrm{NADH}$ oxidation by AAPH. Experiments were performed in a $2 \mathrm{ml}$ final volume containing $50 \mathrm{mM}$ phosphate buffer $\mathrm{pH} 7.4$ or $\mathrm{pH} 6.3,150 \mu \mathrm{M}$ of NADH in the presence or absence of $1 \mathrm{mM}$ TEMPOL.

Table 1. Effects of NADH on egg yolk lecithin (EYL) liposome peroxidation induced by methoxy-AMVN

\begin{tabular}{cc}
\hline NADH $(\mu \mathrm{M})$ & $\begin{array}{c}\text { Peroxidation rate } \\
\left(\mu \mathrm{M} \mathrm{O}_{2} / \mathrm{min}\right)\end{array}$ \\
\hline Control & $10.25 \pm 1.0$ \\
NAD $^{+} 50-500 \mu \mathrm{M}$ & $10.50 \pm 0.5$ \\
NADH 10 $(\mu \mathrm{M})$ & $7.25 \pm 0.75$ \\
NADH $25(\mu \mathrm{M})$ & $3 \pm 0.75$ \\
NADH $50(\mu \mathrm{M})$ & $2.75 \pm 0.5$ \\
NADH 100 $(\mu \mathrm{M})$ & $3.25 \pm 0.5$
\end{tabular}

Oxygen consumption expressed in $\mu \mathrm{M} \mathrm{O} /$ min was measured using an oxygraph and is reported vs NADH concentration. The incubation medium $(2 \mathrm{ml})$ contained $50 \mathrm{mM}$ phosphate buffer $\mathrm{pH}$ 7.4, and $1 \mathrm{mM}$ methoxy-AMVN. Control experiments were performed at $\mathrm{NAD}^{+}$concentration, of 50,100 , and $500 \mu \mathrm{M}$.

peroxyl radical and the carbon center radical), which are both responsible for cell lipid peroxidation (Brault et al., 1985), $\mathrm{NADH}$ could be used as an agent to protect against such oxidation. The effect of NADH or of $\mathrm{PyH}_{2}$ its analogue on the peroxidation of membrane lipids was investigated using EYL liposomes. Experiments were performed using two different peroxyl-generating systems.

The peroxidation of EYL liposomes was studied using peroxyls generated from methoxy-AMVN. Moreover, this study revealed the antioxidant activity of NADH (Table 1), an effect reinforced by the absence of an effect in the NAD ${ }^{+}$control.

The same protective effect of NADH was observed when EYL liposomes were oxidized by tert-butyl peroxyls, as described above (see Fig. 3). We also observed that NADH lipid peroxidation inhibition is not concentration dependent (Fig. 2). This can in part be due to consumption of oxygen by the NAD radical. The interaction between NADH and peroxyl radical, both in solution and in the membrane, reduces lipid peroxidation and at the same time produces superoxide an

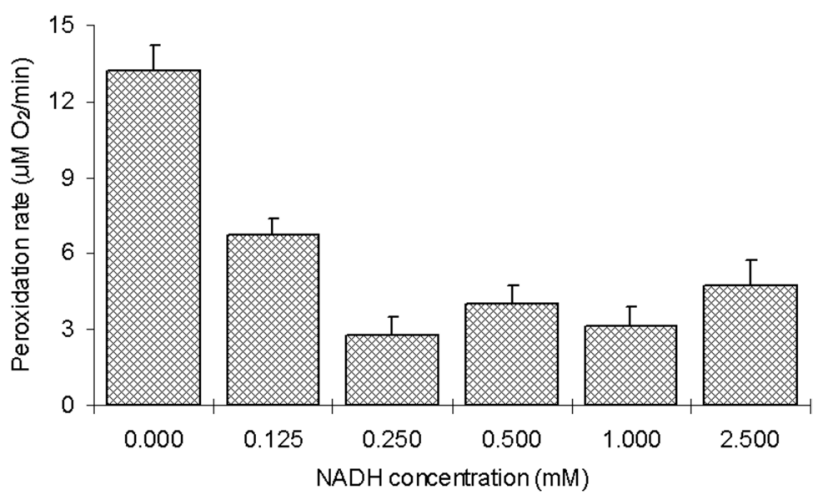

Fig. 2. Effects of $\mathrm{NADH}_{2}$ on egg yolk lecithin (EYL) liposomes peroxidation induced by tert-butyl peroxyls. Oxygen consumption expressed in $\mu \mathrm{M} \mathrm{O}_{2} /$ min was measured using an Oxygraph, and is reported vs $\mathrm{NADH}_{2}$ concentration.

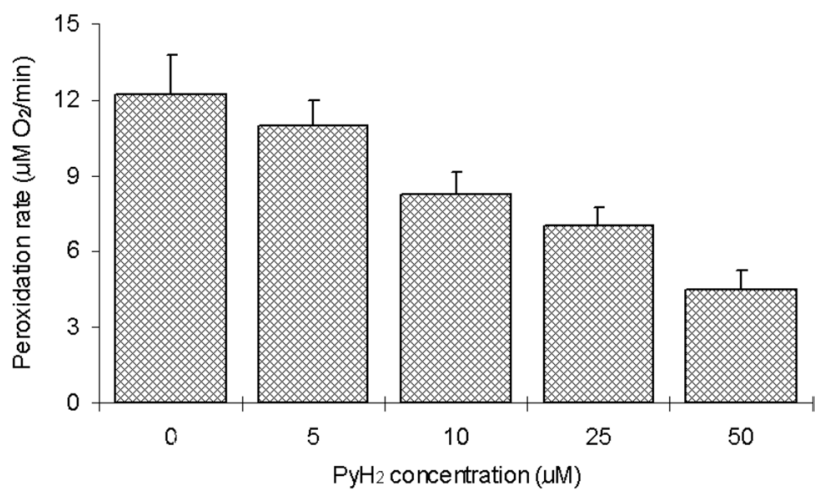

Fig. 3. Effects of $\mathrm{PyH}_{2}$ on egg yolk lecithin (EYL) liposomes peroxidation induced by tert-butyl peroxyls. Oxygen consumption expressed in $\mu \mathrm{M} \mathrm{O}_{2} /$ min was measured using an Oxygraph and is reported vs $\mathrm{PyH}_{2}$ concentration.

anion radical. By employing the same experimental conditions, the antioxidant activity of $\mathrm{PyH}_{2}$ was tested. The reaction rate constant between free radicals of this compound and the free radicals generated by $\mathrm{PyH}_{2}$ is similar to that of NADH (Huyser et al., 1972; You, 1985). The data presented in Fig. 3 indicate a linear relationship between $\mathrm{PyH}_{2}$ inhibition and its concentration. Our data indicate that the hydrophobicity of $\mathrm{PyH}_{2}$ make it a better inhibiter of lipid peroxidation. Presumably, this is due to limited NADH penetration into the lipid bilayer.

Effect of NADH and $\mathrm{PyH}_{2}$ on AAPH mediated DPBF quenching in EYL-liposomes and triton micelles In order to assess whether NADH penetration into the lipid membrane limits its antioxidant activity, the oxidation of DPBF and the effects of NADH and of its analogue on this process were investigated. DPBF is a fluorescent probe, which locates preferentially in the hydrophobic domains of membranes, and after oxidation by singlet oxygen, a peroxyl or an alkoxyl radical DPBF looses its fluorescence (Wozniak et al., 1991; 


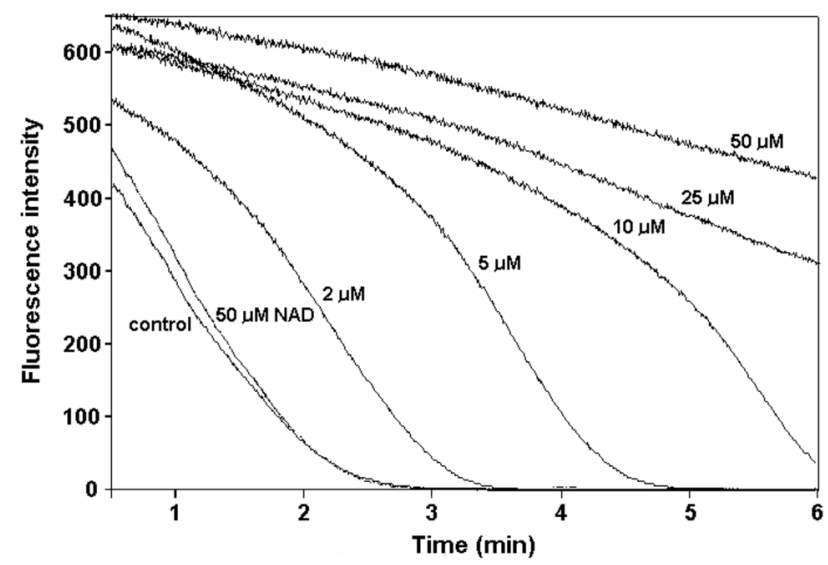

Fig. 4. Effect of NADH on DPBF quenching induced by the thermal decomposition of $6 \mathrm{mM}$ AAPH at $37^{\circ} \mathrm{C}$. DPBF was incorporated into $0.5 \%$ Triton $\mathrm{X}-100$ micelles. The medium consisted of $50 \mathrm{mM}$ phosphate buffer and $5 \mathrm{mM}$ EDTA at $\mathrm{pH}$ 7.4 and NADH at 0 (control), 2, 5, 10, 25, or $50 \mu \mathrm{M}$, as indicated, and NAD at $50 \mu \mathrm{M}$.

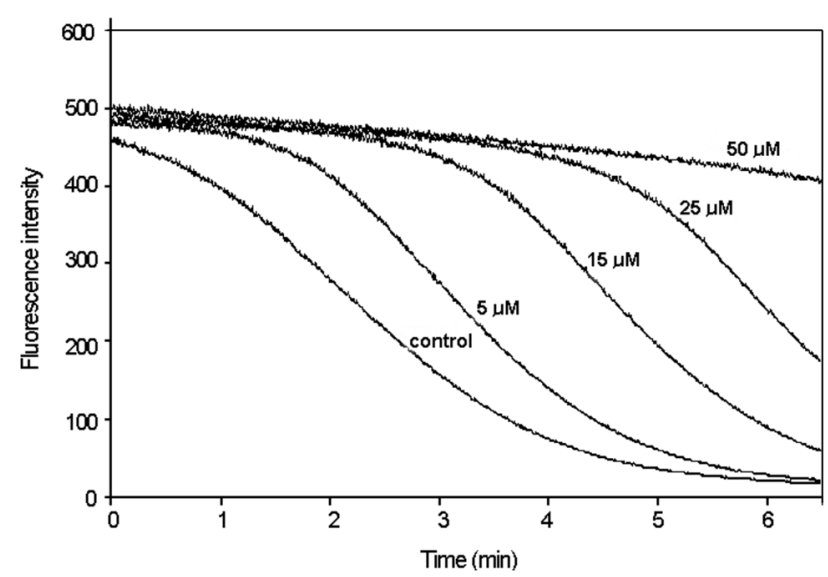

Fig. 5. Effect of NADH on DPBF quenching induced by the thermal decomposition of $6 \mathrm{mM}$ AAPH at $37^{\circ} \mathrm{C}$. DPBF was incorporated into EYL liposomes. The medium consisted of 50 $\mathrm{mM}$ phosphate buffer and $5 \mathrm{mM}$ EDTA at $\mathrm{pH} 7.4$, liposomes (5 $\mathrm{mg}$ of EYL) and NADH at 0 (control), 5, 15, 25, or $50 \mu \mathrm{M}$ as indicated.

Wozniak et al., 1993; Tanfani et al., 1994). We observed that DPBF fluorescence was quenched by peroxyls generated from AAPH, and that this process was more effective in Triton micelles than in EYL liposomes $(350 \pm 23$ vs. $90 \pm 15$ fluorescence units per min) (Fig. 4 and Fig. 5). These data are in agreement with previous observations, which showed that the physical state of a membrane influences DPBF oxidation by ROS. Increased membrane fluidity promotes reaction between DPBF and radicals (Wozniak et al., 1991). NADH was found to prevent DPBF oxidation more effectively in Triton micelles than in liposomes (Fig. 4 and Fig. 5). Moreover, in the case of $\mathrm{PyH}_{2}$, we observed a slightly better protective effect on DPBF quenching in Triton micelles than

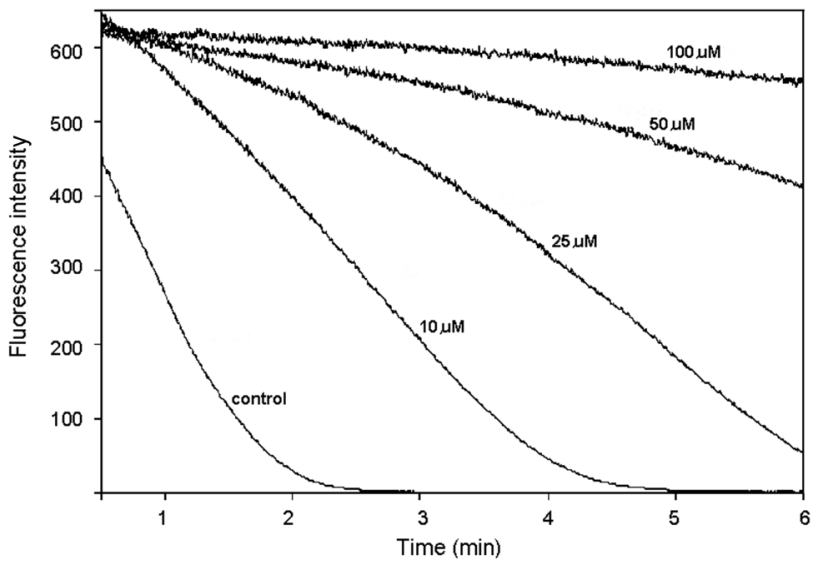

Fig. 6. Effect of $\mathrm{PyH}_{2}$ on $\mathrm{DPBF}$ quenching induced by the thermal decomposition of $6 \mathrm{mM}$ AAPH at $37^{\circ} \mathrm{C}$. DPBF was incorporated into $0.5 \%$ Triton $\mathrm{X}-100$ micelles. The medium contained $50 \mathrm{mM}$ phosphate buffer and $5 \mathrm{mM}$ EDTA at $\mathrm{pH} 7.4$ and $\mathrm{PyH}_{2}$ at 0 (control), 10, 25, 50, or $100 \mu \mathrm{M}$ as indicated.

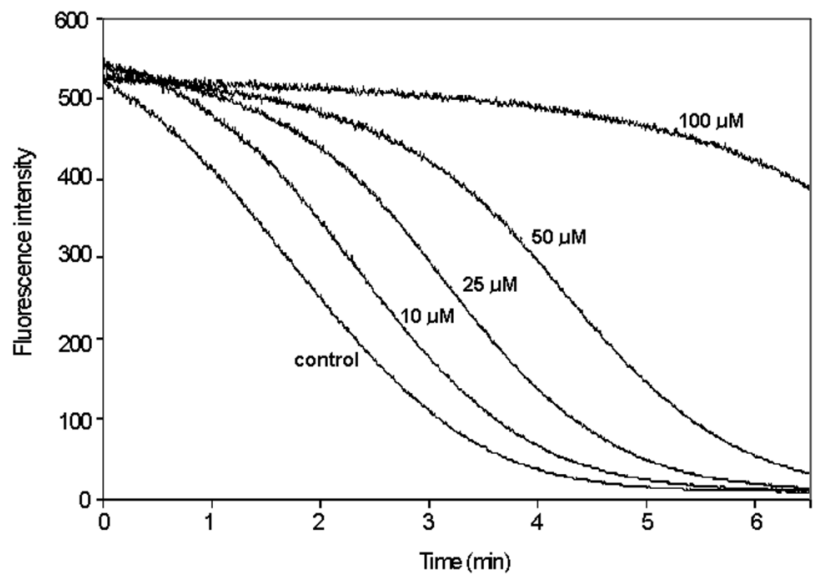

Fig. 7. Effect of $\mathrm{PyH}_{2}$ on DPBF quenching induced by the thermal decomposition of $6 \mathrm{mM}$ AAPH. DPBF was incorporated into EYL liposomes. The medium contained $50 \mathrm{mM}$ phosphate buffer and $5 \mathrm{mM}$ EDTA at pH 7.4, liposomes (5 mg of EYL) and 0 (control), 10, 25, 50, or $100 \mu \mathrm{M}$ of $\mathrm{PyH}_{2}$.

in liposomes (Fig. 6 and Fig. 7).

When we compared the times needed to reduce DPBF fluorescence by $50 \%$, we found it took less then one minute in Triton micelles and $2.13 \mathrm{~min}$ in liposomes (Table 2). The effects of $10 \mu \mathrm{M}$ NADH were stronger than those of $\mathrm{PyH}_{2}$ in liposomes and Triton micelles when AAPH was used as a radical source. Moreover, $\mathrm{PyH}_{2}$ became more effective when hydrophobic metoxy-AMVN or tert-butyl hydroperoxide with cytochrome c were used as radical sources (Table 2).

A stronger antioxidant activity of $\mathrm{NADH}$, as compared to $\mathrm{PyH}_{2}$ (Fig. 4, Fig. 6 and Table 2), was observed when the hydrophilic azo-initiator (AAPH) was applied in Triton micelles. This was attributed to the ability of NADH to react directly with peroxyl radicals in the aqueous phase, whereas 
Table 2. The effect of NADH and $\mathrm{PyH}_{2}$ on DPBF fluorescence bleaching

\begin{tabular}{cccc}
\hline & Control & $10 \mu \mathrm{M} \mathrm{NADH}$ & $10 \mu \mathrm{M} \mathrm{PyH}_{2}$ \\
\hline Triton + AAPH & $0.80 \pm 0.12$ & $4.37 \pm 0.15$ & $1.85 \pm 0.21$ \\
Liposomes + AAPH & $2.13 \pm 0.29$ & $3.70 \pm 0.50$ & $2.35 \pm 0.21$ \\
Liposomes + Bu'OOH + cyt. c & $0.73 \pm 0.10$ & $0.78 \pm 0.05$ & $0.90 \pm 0.03$ \\
Liposomes + methoxy-AMVN & $2.38 \pm 0.13$ & $3.05 \pm 0.07$ & $3.45 \pm 0.19$ \\
\hline
\end{tabular}

DPBF was incorporated into egg yolk lecithin liposomes or $0.5 \%$ Triton X-100 micelles. The medium consisted of $50 \mathrm{mM}$ phosphate buffer and $5 \mathrm{mM}$ EDTA at $\mathrm{pH} 7.4$ and $10 \mu \mathrm{M}$ NADH or $10 \mu \mathrm{M} \mathrm{PyH}$. AAPH and methoxy-AMVN were at concentrations of $6 \mathrm{mM}$, cytochrome c at $4.2 \mu \mathrm{M}$, and $\mathrm{Bu}{ }^{t} \mathrm{OOH}$ at $3.6 \mathrm{mM}$. The data show the time (min) necessary to reduce DPBF fluorescence by $50 \%$.

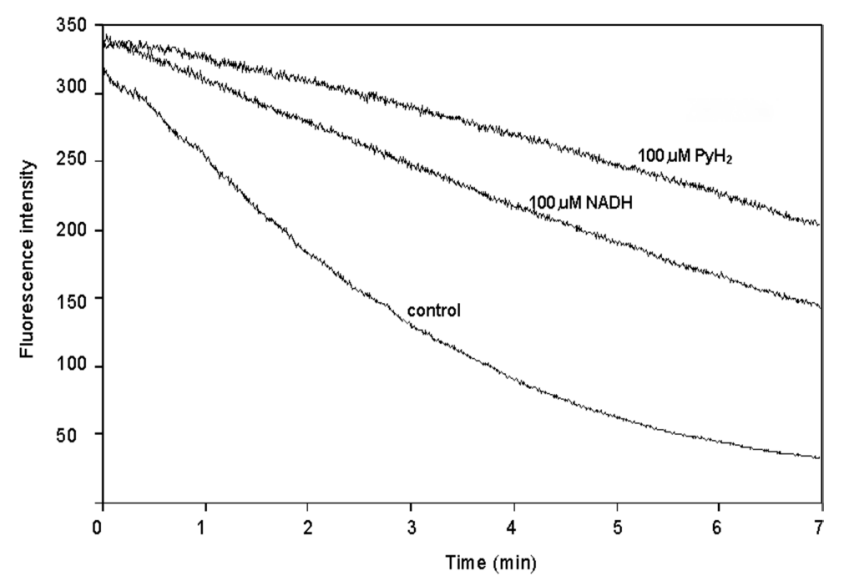

Fig. 8. Effects of $\mathrm{NADH}$ or $\mathrm{PyH}_{2}$ on DPBF quenching induced by the thermal decomposition of $6 \mathrm{mM}$ methoxy-AMVN. DPBF was incorporated into EYL liposomes. The medium contained 50 $\mathrm{mM}$ phosphate buffer and $5 \mathrm{mM}$ EDTA at $\mathrm{pH} 7.4$, liposomes (5 $\mathrm{mg}$ of EYL) and 0 (control), or $100 \mu \mathrm{M}$ concentrations of $\mathrm{NADH}$ or $\mathrm{PyH}_{2}$.

$\mathrm{PyH}_{2}$ reacts only in the hydrophobic membrane. Moreover, this results shows that NADH activity is related to its ability to protect DPBF from oxidation in membranes. On the other hand, when a hydrophobic azo compound (methoxy-AMVN) was used, $\mathrm{PyH}_{2}$ protected DPBF in liposomes against oxidation more effectively than NADH (Fig. 8). In this case, the $\mathrm{PyH}_{2}$ was probably more effective because it better penetrates the membrane, the only place where methoxyAMVN generates peroxyl radicals (Noguchi et al., 1998). Our results indicate that the penetration of NADH into the hydrophobic domain of liposomes, where DPBF is located, is limited. Thus, its antioxidant activity is more effective the micelles. Several results show that the reactivities of hydrophobic compounds located in biological membranes with compounds located either outside or inside the membranes depends on the physical state of the membrane and the localization of the compound concerned. For example, Takahashi, et al. (1989) reported that the antioxidant activity of the nitroxide radical attached to a fatty acid, reduces with membrane penetration depth. The different effects of NADH and $\mathrm{PyH}_{2}$ could be partially explained from the chemistry of $\mathrm{PyH}_{2}$. When it loses hydrogen, a newly formed $\mathrm{PyH}$ radical does not react with dioxygen like the NAD radical, but it rapidly loses another hydrogen, to form an aromatic pyridine (2,6-dimethyl-3,5-dicarbethoxypyridine) (Huyser et al., 1972). Thus, the antioxidant action of $\mathrm{PyH}_{2}$ does not lead to a rise in superoxide and related radicals. Because DPBF does not react with superoxide, its oxidation should not be influenced by the $\mathrm{O}_{2}^{-}$formed during $\mathrm{NADH}$ oxidation. This confirms the supposition that observed differences in the antioxidant activities of NADH and its analogue are mainly due to their distinct hydrophobicities.

It was previously reported that $200 \mathrm{M}$ NADH is able to inhibit lipid peroxidation in submitochondrial particles, although at lower concentrations it stimulates this process (Glinn et al., 1991). Moreover, Cavallini et al. (1983) observed that NADH and NADPH inhibit the lipid peroxidations induced by cumene hydroperoxide, linolenic acid hydroperoxide or peroxidized phosphatidylcholine in rat liver microsomes. They concluded that this inhibition was not due to a direct antioxidant effect of NADH and NADPH. It has been also shown that a reconstituted mixed-function oxidase from liver microsomes can catalyze the NADPH dependent reduction of 13-hydroperoxyl-9,11 octadecadienoic acid (Lindstrom and Aust, 1984). Moreover, it was suggested recently that antioxidant function of $\mathrm{NAD}(\mathrm{P}) \mathrm{H}$ in living cells is partially related to its direct reaction with various radical species (Kirsch and De Groot, 2001).

$\mathrm{NAD}(\mathrm{P}) \mathrm{H}$ is involved in many enzymatic reactions in living cells. Thus, it is difficult to investigate its antioxidant properties in vivo. In order to avoid any superfluous enzymatic reactions in which NADH could participate, we employed EYL liposomes and Triton micelles in vitro to investigate whether NADH is able to protect lipids against oxidation.

In summary, the data obtained support the hypothesis that nicotinamide nucleotides act as direct antioxidants, even in nonenzymatic reactions. However, the effectiveness of such action depends on hydrophobicity of the reaction environment. Here, we postulate that changes in the NADH / NAD ${ }^{+}$ratio can significantly influence the antioxidant potential directed against peroxyl radical cytotoxicity in living cells.

Acknowledgments This work was supported by KBN grant 4 P05A 125 19. The authors thank Dr. Elisabetta Damiani and Dr. Jerzy Nowak for helpful discussions. 


\section{References}

Batzri, S. and Korn, E. D. (1973) Single bilayer liposomes prepared without sonication. Biochim. Biophys. Acta 298, 10151019.

Bodaness, R. S. and Chan, P. C. (1977) Singlet oxygen as a mediator in the hematoporphyrin-catalyzed photooxidation of NADPH to $\mathrm{NADP}^{+}$in deuterium oxide. J. Biol. Chem. 252, 8554-8560.

Brault, D., Neta, P. and Patterson, L. K. (1985) The lipid peroxidation model for halogenated hydrocarbon toxicity. Kinetics of peroxyl radical processes involving fatty acids and $\mathrm{Fe}(\mathrm{III})$ porphyrins. Chem. Biol. Interact. 54, 289-297.

Carlson, B. W., Miller, L. L., Neta, P. and Grodkowski, J. (1984) Oxidation of NADH involving rate-limiting one-electron transfer. J. Am. Chem. Soc. 106, 7233-7239.

Cavallini, L., Valente, M. and Bindoli, A. (1983) NADH and NADPH inhibit lipid peroxidation promoted by hydroperoxides in rat liver microsomes. Biochim. Biophys. Acta 752, 339-345.

Chan, P. C. and Bielski, B. H. (1974) Enzyme-catalyzed free radical reactions with nicotinamide adenine nucleotides. II. Lactate dehydrogenase-catalyzed oxidation of reduced nicotinamide adenine dinucleotide by superoxide radicals generated by xanthine oxidase. J. Biol. Chem. 249, 1317-1319.

Chan, P. C. and Bielski, B. H. (1980) Glyceraldehyde-3-phosphate dehydrogenase-catalyzed chain oxidation of reduced nicotinamide adenine dinucleotide by perhydroxyl radicals. $J$. Biol. Chem. 255, 874-876.

Chateauneuf, J., Lusztyk, J. and Ingold, K. U. (1988) Absolute rate constants for the reactions of some carbon-centered radicals with 2,2,6,6-tetramethylpiperidine-N-oxyl. J. Org. Chem. 53, 1629-1632.

Forni, L. G. and Willson, R. L. (1986a) Thiyl and phenoxyl free radicals and NADH. Direct observation of one-electron oxidation. Biochem. J. 240, 897-903.

Forni, L. G. and Willson, R. L. (1986b) Thiyl free radicals and the oxidation of ferrocytochrome c. Direct observation of coupled hydrogen-atom- and electron-transfer reactions. Biochem. J. 240, 905-907.

Gieseg, S., Duggan, S. and Gebicki, J. M. (2000) Peroxidation of proteins before lipids in U937 cells exposed to peroxyl radicals. Biochem. J. 350, 215-218.

Glinn, M., Ernster, L. and Lee, C. P. (1991) Initiation of lipid peroxidation in submitochondrial particles: Effect of respiratory inhibitors. Arch. Biochem. Biophys. 290, 57-65.

Huyser, E. S., Harmonyand, J. A. K. and McMillian, F. L. (1972) Peroxide oxidation of dihydropyridine derivatives. J. Am. Chem. Soc. 94, 3176-3180.

Kirsch, M. and De Groot, H. (2001) NAD(P)H, a directly operating antioxidant? FASEB J. 15, 1569-1574.
Lieber, C. S. (1997) Role of oxidative stress and antioxidant therapy in alcoholic and nonalcoholic liver diseases. $A d v$. Pharmacol. 38, 601-628.

Lindstrom, T. D. and Aust, S. D. (1984) Studies on cytochrome P450-dependent lipid hydroperoxide reduction. Arch. Biochem. Biophys. 233, 80-87.

Niki, E. (1990) Free radical initiators as source of water- or lipidsoluble peroxyl radicals. Methods Enzymol. 186, 100-108.

Noguchi, N., Yamashita, H., Gotoh, N., Yamamoto, Y., Numano, R. and Niki, E. (1998) 2,2'-Azobis (4-methoxy-2,4dimethylvaleronitrile), a new lipid-soluble azo initiator: application to oxidations of lipids and low-density lipoprotein in solution and in aqueous dispersions. Free Radic. Biol. Med. 24, 259-268.

Sahlin, K., Harris, R. C., Nylind, B. and Hultman, E. (1976) Lactate content and $\mathrm{pH}$ in muscle obtained after dynamic exercise. Pflug. Archiv. Eur. J. Physiol. 367, 143-149.

Samuni, A., Krishna, C. M., Mitchell, J. B., Collins, C. R. and Russo, A. (1990) Superoxide reaction with nitroxides. Free Radic. Res. Commun. 9, 241-249.

Schantz, P. G. (1986) Plasticity of human skeletal muscle with special reference to effects of physical training on enzyme levels of the NADH shuttles and phenotypic expression of slow and fast myofibrillar proteins. Acta Physiol. Scand. Suppl. 558, $1-62$.

Takahashi, M., Tsuchiya, J. and Niki, E. (1989) Scavenging of radicals by vitamin $\mathrm{E}$ in the membranes as studied by spin labeling. J. Am. Chem. Soc. 111, 6350-6353.

Tanfani, F., Fiorini, R., Tartaglini, E., Kantar, A., Wozniak, M., Antosiewicz, J. and Bertoli, E. (1994) A sensitive detection of neutrophil activation by fluorescence quenching of membrane inserted singlet oxygen probe. Biochem. Mol. Biol. Int. 32, 1093-1099.

Tischler, M. E., Friedrichs, D., Coll, K. and Williamson, J. R. (1977) Pyridine nucleotide distributions and enzyme mass action ratios in hepatocytes from fed and starved rats. Arch. Biochem. Biophys. 184, 222-236.

Wozniak, M., Nowak, J., Antosiewicz, J., Greci, L., Damiani, E., Tanfani, F. and Bertoli, E., (1993) Diphenylisobenzofuran (DPBF) follows the dynamics of ROO to RO and C-centered radicals in microsomal membranes. Biochem. Soc. Trans. 21, 85.

Wozniak, M., Tanfani, F., Bertoli, E., Zolese, G. and Antosiewicz, J. (1991) A new fluorescence method to detect singlet oxygen inside phospholipid model membranes. Biochim. Biophys. Acta 1082, 94-100.

You, K. S. (1985) Stereospecificity for nicotinamide nucleotides in enzymatic and chemical hydride transfer reactions. CRC Crit. Rev. Biochem. 17, 313-451. 